\title{
The Research About Remote Transmit Manage Unit Which Is Based On ModBus Protocol
}

\author{
Guojun WANG \\ Langfang Yanjing Polytechnic \\ Langfang, China.P.R \\ e-mail: wangyy2007@126.com
}

\begin{abstract}
ModBus Protocol is researched and developed by MODICOM company which owns its copyright.The research about Remote Transmit Manage Unit applies the opened ModBus protocol. The text of protocol itself is very simple and can be easily obtained.The protocol is surpported by both domestic manufacturers and international manufacturers.According to the device which we will research, we apply a mini-subsets of Modbus protocol and don't realize all content
\end{abstract}

Keywords-Modbus; primary device; alternating current;direct current

\section{I.INTRODUCTION OF MODBUS PROTOCOL}

Modbus protocol is a general language used in electro-controller. By using the protocol, controllers can communicate with each other, so do other devices ( such as Ethernet) with network.It has already become a general industrial standard. Since it has come into being, control equipment coming from different manufacturer can be connected with industrial network. By industrial network we can monitor all control equipment at the same time.

Modbus protocol defined a message structure which can be accepted by controllers, no matter what kinds of network we communicate with this message struncture.It describes the process that controllers request and access other devices ,including how to react to other device's request, monitor errors and mark it.The protocol defined the pattern of message field and the contents of the public format.

When we communicate with each other through Modbus network,the protocol determines that every controller must know their device addresses, recognize the message according to their addresses and decide how to react . If device needs to be responded,the controller will generate reactive information and transmit the information through Modbus protocol.

when we communicate with each other through other networks, the message transformation of the Modbus protocol becomes a part of the frame or packet structure.The transformation expands the method that solves node address、 route and error checking on the basis of specfic network.

\section{II.Transmitting Message By ModBus NetworK}

Standard Modbus interface is RS-232C or serial interface,that defines the number of needle、 type of cable、 the number of singal-bit、 baud rate and parity check.The controller can form network either directly or with modem.
Controller communication uses the technology of principal and subordinate,that is to say, only primary device can start transmission(query).Other devices( slave device) make an appropriate response according to the data that is provided by primary device. Typical primary devices may be host or remote transmit manage unit.

Primary device can communicate with not only one individual slave device, but also all slave devices by broadcast mode.when it communicates with one, slave device returns message as a response.when it communicates with all, no one replys to primary device.Modbus protocol describes the format of primary device inquiry:device address(or broadcast address)、 function source、 all data to be sent and domain for checking error .

The message that slave device returned is also made up of Modbus protocol. It includes domain that confirms how to act any data to be returned and domain for checking error.Slave device will create message and send it as responded message, when there are data errors for slave device to receive messages or slave device can not execute the command that primary device sends.

\section{III.THEORY INTRODUCTION}

Remote Transmit Manage Unit uses 32 bit ARM chip and uCOS operation system ( real time operation system) .It unites many technologies together, such as Ethernet communication 、 read analogue input 、 read analogue output、switch input、switch output wireless communication and so on; it is a highly intergrated office terminal that is specially designed for data acquisition、 data storage、data control and long-distance communication in industrial process ; it has the capacity of three-phase alternating sampling, uses special three-phase multifunctional chip for calculation, highlights precision energy accumulation and measurement precision and meets requirements of general oil field for detailed management 、 energy-saving and emission-reduction. So it can be applied to auto-projects, for example, motor-pumped well telemetering valculating room and waterflooding room.

\section{A..MODBUS-RTU Information Frame}

Regardless of what kind of network transmission model,the information of ModBus transmits with frame. Because every frame has clear start and end, receiving equipment can read address at the beginning of the 
information and confirm the addressed device(when broadcasting,to all devices).

\begin{tabular}{|c|c|c|}
\hline Start & Address & Function \\
\hline T1-T2-T3-T4 & 8bit & 8bit \\
\hline Data & Crc & End \\
\hline N×8 bit & 16bit & T1-T2-T3-T4 \\
\hline
\end{tabular}

Fig. 1 Modbus RTU Frame Format

\section{B.TCP Information-Frame}

The mode involved with TCP Information-Frame is used as the application layer of TCP protocol. The frame has no start, end character or silent period.Compared with RTU mode,it adds 6 bytes before RTU and cuts down the check domain of CRC.

\begin{tabular}{|l|l|l|}
\hline $\begin{array}{l}\text { Transaction } \\
\text { Identifier }\end{array}$ & $\begin{array}{l}\text { Protocol } \\
\text { Identifier }\end{array}$ & $\begin{array}{l}\text { Length } \\
\text { Field }\end{array}$ \\
\hline 2 bytes & 2bytes & 2bytes \\
\hline Unit Identifier & $\begin{array}{l}\text { Function } \\
\text { code }\end{array}$ & $\begin{array}{l}\text { Data } \\
\text { bytes }\end{array}$ \\
\hline 1byte & 1byte & N bytes \\
\hline
\end{tabular}

Fig.2 Modbus TCP Frame Format

\section{IV.The RESEARCh ABOUt REMOte TRANSMIT MANAge Unit BASED ON MODBus PROTOCOL}

\section{A. Basic Design Concept}

Remote Transmit Manage Unit are provided with 8-way analog input 4-way switch/Pulse quantity / frequency input、2-way analog quantity and 5-way switching relay output, can be applied to different industries by inputing different sensors,such as the monitored control system of oil field v the monitored control system of angricuture 、 the monitored control system of electric power、 the monitored control system of water conservancy.

The external sensors of the unit are power 、 temperature、 rate of flow, rotation rate、 toxic gas air humidity dust content sensors separately, conforming to industry-standard with the range of current ( $4 \sim 20 \mathrm{~mA})$ or the range of voltage $(0 \sim 5 \mathrm{~V})$.

The unit is specially designed to monitor protection for high and low voltage v network system and power; and it is applied to such occasions that need three-phase electricity and remote control, for example, chemical industry 、 transferring-station of oil field w water wells and station of lift pump.

At the same time the unit is the management device of oil well monitoring center that is applied to the monitoring system of pumping unit, collecting the real-time parameters about tubing pressure of oil field scene、 casing pressure、 well head temperature and motor protection unit.

The unit provided with various communication interfaces: RS232 interface、RS485 interface、wireless
interface、GPRS interface、 Ethernet interface (contains one TCP Server port and one TCP Client port) can form network to realize a variety of remote line network or wireless network.According to the situation of actual application,except GPRS port and Ethernet TCP Server port, we can appoint one active port among other ports. The active port is equal to the port that Modbus protocol sets up to query.

\section{B. Feature of Function}

- Three-meter method measures exactly three-phase alternating voltage、current、usable power、deaktive Leistung、frequency , power factor、 residual current residual voltage 、 forward-order current 、 negative-sequence current and so on, having a precision up to $0.5 \%$.

- The unit having free-running switch output with 5can be applied to remote control、tripping operation or alarm.

- The unit having switch input with 4also can be applied to pulsed quantity and frequency number.

- The unit having isolated direct current sampling with 8 can connect various sensors or transducer that conforms to industry-standard.

- The unit having RS232 interface with 1supports MODBUS-RTU protocol.

- The unit having RS485 interface with 1supports MODBUS-RTU protocol.

- The unit having 433 wireless interface with 1 connects network easily and accords to European standard system of ETSI(EN200-220-1) 、FCC(15.247 and 15.249)and ARIB STD-T67. Its design conforms to wireless control and supports MODBUS-RTU protocol without applying for the licence of using frequency .

- The unit having GPRS/CDMA wireless interface with 1can connect wireless network with long distance and wide range and it supports MODBUS-RTU protocol.

- The unit having 100Mbps Ethernet interface with 1can communicate with various interfaces unit to easily realize the connection with wired fiber or 3G/4G/5G wireless network and it supports MODBUS-TCP protocol.

- The unit can gather displacement、 loading、current、 power with other devices, making the diagrammatic drawing of loading-displacement the diagrammatic drawing of current-displacement the diagrammatic drawing of power-displacement.

\section{CONClusion AND ReVElation}

The concept of remote transmit manage unit is based on the application of MODBUS protocol. It is a preliminary study. In fact, during the process of operation we will probably meet a lot of troubles, for example, data encode、 data compression v the design of new interface and so on. We hope to improve and perfect it in the future. 


\section{REFERENCES}

[1]Alexander Bormann,Ingo Hilgenkamp. Industrial Networks Ethernet Communication for Automation Applications. National defence of Industry Press,BeiJing,2011.

[2] Zanesville, Ohio. Articles on Industrial Ethernet, Including: Modbus, Profinet, Avionics Full-Duplex Switched Ethernet, List of Automation Protocols, Ethernet Powerlink. Hephaestus Books,US 2011.

[3] Steve Mackay,Edwin Wright,Deon Reynders,John Park.Practical Industrial Data Networks: Design,Installation and Troubleshooting. Newnes (an imprint of Butterworth-Heinemann Ltd ) ,US,2004.
[4]Rong Hua.FROM Modbus to Transparent ready---The Electric Industrial Network of Schneider Protocol 、 design 、 installation and application. China Machine Press,BeiJing,2009.

[5]Jin Kuan Wang,Shu Hua Ma,Yu Chuan Wu.The Technology of Indurstrial Network. Beijing University of Posts and Telecommunications press,BeiJing,2007.

[6] Axelson.The Technology of Communications Design about embedded Ethernet and Internet. Beijing University of Aeronautics and Astronautics Press, BeiJing. 2006. 